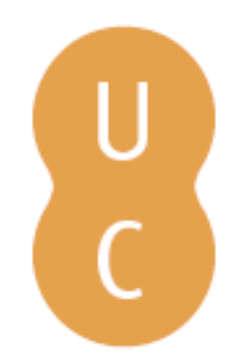

\title{
pommalina
}

\section{La Archivistica y las disciplinas informativas documentales: retos y cuestionamientos epistemológicos}

\author{
Autor(es): $\quad$ Rendón Rojas, Miguel Ángel; Cruz Domínguez, Silvana Elisa \\ Publicado por: Imprensa da Universidade de Coimbra \\ URL \\ persistente: $\quad$ URI:http://hdl.handle.net/10316.2/31891 \\ DOI: $\quad$ DOI:http://dx.doi.org/10.14195/978-989-26-0319-3_17 \\ Accessed : $\quad$ 26-Apr-2023 16:22:17
}

A navegação consulta e descarregamento dos títulos inseridos nas Bibliotecas Digitais UC Digitalis, UC Pombalina e UC Impactum, pressupõem a aceitação plena e sem reservas dos Termos e Condições de Uso destas Bibliotecas Digitais, disponíveis em https://digitalis.uc.pt/pt-pt/termos.

Conforme exposto nos referidos Termos e Condições de Uso, o descarregamento de títulos de acesso restrito requer uma licença válida de autorização devendo o utilizador aceder ao(s) documento(s) a partir de um endereço de IP da instituição detentora da supramencionada licença.

Ao utilizador é apenas permitido o descarregamento para uso pessoal, pelo que o emprego do(s) título(s) descarregado(s) para outro fim, designadamente comercial, carece de autorização do respetivo autor ou editor da obra.

Na medida em que todas as obras da UC Digitalis se encontram protegidas pelo Código do Direito de Autor e Direitos Conexos e demais legislação aplicável, toda a cópia, parcial ou total, deste documento, nos casos em que é legalmente admitida, deverá conter ou fazer-se acompanhar por este aviso.

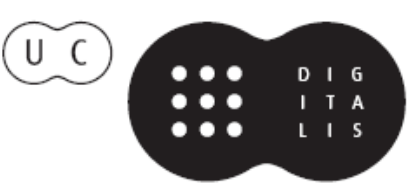


Maria Manuel Borges

Elias Sanz Casado

Coordenação

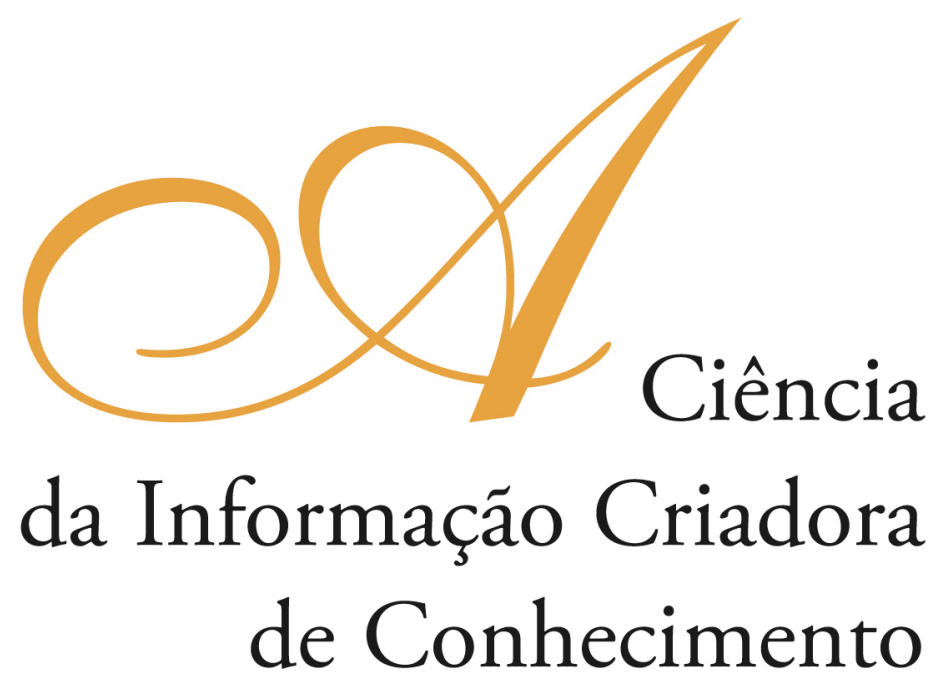

Vol. I

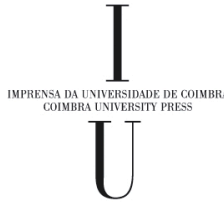

- COIMBRA 2009 


\title{
La Archivistica y las Disciplinas Informativas
}

Documentales: Retos y Cuestionamientos Epistemológicos

\author{
Miguel Ángel Rendón Rojas \\ Universidad Nacional Autónoma de México (México) \\ Silvana Elisa Cruz Domínguez \\ Universidad Autónoma del Estado de México (México)
}

\section{Resumen}

En su devenir histórico, la Archivística ha pasado por diversos avatares: durante el siglo XIX, fue una ciencia auxiliar de la Historia; a mediados del siglo XX se convirtió en auxiliar de la Administración; después reclamó su estatuto como ciencia autónoma; y finalmente ahora, se le asocia a la Ciencia de la Información o de la Documentación. Asimismo el acelerado desarrollo de las tecnologías de la información y la comunicación, la aparición de los documentos electrónicos y finalmente el pensamiento filosófico llamado postmodernismo pusieron en jaque los principios fundamentales de la archivística. Aún más, se han llegado a cuestionar sus principios teóricos, aquellos que le dan el sustento para denominarse ciencia. Ante tal situación, es necesario reflexionar sobre los retos y cuestionamientos epistemológicos que afronta la Archivística como ciencia; los presupuestos y consecuencias de la armonización; los cambios en su estructura teórica que son necesarios realizar ante los nuevos fenómenos, procesos y contextos que se presentan en la actualidad.

\begin{abstract}
Como una posible respuesta ante los cambios experimentados por la Archivística, en la década de los ochenta del siglo XX, se propuso la armonización, la cual fue derivada del acercamiento propiciado por el desarrollo de la tecnología, entre las disciplinas que tienen en común el proceso informativo documental: la Bibliotecología, la Archivística y la Documentación. El postulado esencial de la armonización era la enseñanza de la Archivística en programas multidisciplinarios, ahora la Archivística no estaría directamente vinculada a la Administración o a la Historia, sino a la Documentación o a la Ciencia de la Información.

Desde hace varias décadas, cuando iniciaba el desarrollo de las tecnologías de información, empezaron a surgir abundantes artículos especializados que versaban sobre el futuro de la Archivística. Varios teóricos han cuestionado sus principios básicos. Por ejemplo, Terry Cook ha disertado en distintas ocasiones acerca de la reflexión a la que nos deben de conducir los cambios sustanciales de las sociedades, en especial, invita al profundo razonamiento sobre las transformaciones suscitadas por las tecnologías de la información y de la comunicación ${ }^{1}$. (Cook, 1997, 2000)
\end{abstract}

\footnotetext{
${ }^{1}$ Cook refiere que la postura de Jenkinson se debía a que laboraba en un archivo de la Edad Media, con fondos cerrados.
} 
Pese a la importancia del tema y la variedad de trabajos escritos, el resultado que tenemos hasta el momento es que aún no existe acuerdo, continúa el debate. Sin embargo es necesario reconocer que esa situación no es privativa de la Archivística, sino que todas las disciplinas involucradas en los procesos informativo-documentales se encuentran en circunstancias similares, dos de ellas: la Bibliotecología y la Documentación, parecen lograr algunas alianzas, no obstante pervive la discusión sobre los límites de sus fronteras.

Como ya hemos mencionado, la Archivística desde su nacimiento ha estado profundamente vinculada a otras áreas del conocimiento. En sus inicios, la Archivística o Archivología, como algunos autores prefieren llamarla, sólo era una disciplina empírica, que era practicada por los administradores de los distintos gobiernos. La literatura que se publicaba al respecto sólo versaba sobre la praxis archivística; en ese tiempo, la disciplina se debía únicamente a la gestión gubernamental.

Durante el siglo XIX, se formularon los principios de procedencia y de orden original, con objeto de darle sustento teórico a la práctica archivística. Era la búsqueda de todas las ciencias, recordemos que primaba la filosofía positivista, la cual exigía la existencia de leyes inmutables y universales. Los principios pronto fueron aceptados y enarbolados como bandera científica de la disciplina.

$\mathrm{Al}$ paso del tiempo, estos principios se han enriquecido para dar solidez a esta área del conocimiento. En primer lugar, la palabra archivo adquirió una doble dimensión, la tradicional de lugar donde se guardan los documentos y otra que responde al nuevo concepto de archivo, acuñado desde fines del siglo XIX, como un conjunto orgánico de documentos. (Fuster Ruíz, 1999) Desde entonces los conceptos sobre archivo se han multiplicado de manera exponencial, pero todos rescatan más o menos la esencia de que los documentos son producidos dentro de la actividad administrativa. Este nuevo concepto dio lugar a su vez a un cambio de nombre de la disciplina, (en especial entre los archivistas europeos) la Archivonomía o Archivología ahora sería llamada Archivística, y se estableció que el objeto de estudio de esta área del conocimiento son los archivos y su método estaría compuesto por los principios de procedencia y de orden original.

Será también durante el siglo XIX, que los documentos de archivo fueron reconocidos como fuentes invaluables de la historia, además de las seculares funciones jurídicas y administrativas que les habían caracterizado hasta entonces. Durante ese tiempo y hasta bien entrada la segunda mitad del siglo XX, la archivística estuvo estrechamente vinculada a la Historia. La organización de los fondos documentales se hacía de acuerdo con los intereses que entonces tenía esta área del conocimiento, los funcionarios de los archivos eran por lo general historiadores y su función consistía en preservar celosamente la documentación a su cuidado y elaborar catálogos de los "ramos" documentales de su propio interés. Fue en ese momento que la Archivística se instituyó como una materia de estudios, pero se estudiaba primordialmente la Diplomática y la Historia de las instituciones, era el tiempo de los tesoros documentales.

Este acercamiento entre la Historia y la Archivística contribuyó a detener temporalmente el desarrollo de la ciencia, pues influyó en los archivistas de varias partes del orbe para aceptar el nuevo concepto de archivo, debido a que la mayoría de ellos estaban concentrados sólo en la documentación histórica. Aún hoy, la formación que se exige para ocupar el puesto de archivista -por lo menos en nuestro país- es el 
grado de historiador. Aunque la renuencia empieza a diluirse y parece haber mayor aceptación a percibir los archivos de forma integral.

Hacia la década de 1950 surgió una nueva teoría: la de "Administración de Documentos”. Este nuevo postulado establecía que no toda la documentación debía conservarse y que los documentos debían gestionarse, para decidir su permanencia o destrucción. (Shellenberg, 1987) Este hecho cimbraría las bases de la Archivonomía tradicional y marcaría un verdadero hito en la teoría y prácticas archivísticas a nivel internacional. También provocó un acalorado debate entre el teórico estadounidense Shellenberg y el archivista inglés Sir Hillary Jenkinson; mientras el primero presentaba como argumento para respaldar su teoría de Records Managements, la inmensa cantidad de documentos producidos por la administración pública, el segundo defendía el carácter probatorio de los documentos, decía que éstos son pruebas de los actos y transacciones de las instituciones y que después de su creación no se debía permitir ninguna selección, pues su carácter imparcial de prueba se vería socavado y se violarían los principios fundamentales de los archivos, los cuales fueron establecidos por el manual holandés. (Cook, 1997) Ambos autores influyeron en los archivistas de todo el mundo, por ello la discusión aún sigue vigente en ciertos ámbitos. ${ }^{2}$

Esta nueva visión de la Archivística redundaría en el retorno de ésta a la administración pública, recordemos que en el período anterior se había volcado hacia la historia. La problemática que aquejaba a los archivos administrativos propició el surgimiento de una nueva teoría: la de las edades de los documentos, según la cual, los documentos nacen, viven y mueren o resucitan (en caso de ser seleccionados para su permanencia). La enseńanza de la Archivística, durante este periodo se dividía en: archivística tradicional y administración de documentos.

En 1992, los archivistas canadienses propusieron una nueva Archivística, la Archivística Integrada, es decir, que no se consideraran disciplinas distintas la Administración o Gestión de Documentos y la Archivística, sino que la primera debía incorporarse a los fundamentos teóricos de la segunda. La fusión de ambas teorías fue aceptada de manera paulatina.

La mayoría de los teóricos consideran que la Archivística es una disciplina en desarrollo o bien una ciencia en desarrollo debido a la crisis por la que está pasando. Por ejemplo, Theo Thomassen se refiere a la archivística "no en el sentido estricto de la ciencia física, sino como un equivalente a la expresión más amplia de la ciencia Wissenschaft: como el término general de una disciplina científica”. El autor utiliza como argumento la obra clásica de Kuhn, en la que un paradigma es un logro científico reconocido universalmente, el cual durante un tiempo, proporciona el modelo explicativo de las disciplinas científicas en una fase específica de su desarrollo y define sus fundamentos. Así, declara que el paradigma clásico de la ciencia archivística no es un paradigma eterno, que el paradigma fue establecido por la publicación del manual holandés, lo que marcó el fin de una revolución científica, una revolución que integró diferentes conceptos y técnicas tomadas de la práctica diplomática y administrativa decimonónicas, en el que hubo acuerdo en el ámbito archivístico durante casi un

\footnotetext{
${ }^{2}$ Cook refiere que la postura de Jenkinson se debía a que laboraba en un archivo de la Edad Media, con fondos cerrados.
} 
siglo, a esta etapa la llama preparadigmática y a la siguiente, - es decir la actual- la de la revolución científica, la cual dio inicio con el desarrollo de las tecnologías de comunicación e información. (Thomassen, 1997)

Como consecuencia de la mencionada idea de armonización, los organismos internacionales de la información auspiciados por la UNESCO, como la IFLA, el CIA y la FID, plantearon la posibilidad de crear un tronco común en las ciencias de la información, es decir, encontrar los puntos comunes de las tradicionales ciencias de la información: archivística, bibliotecología y documentación. Se encontró que todas ellas comparten el mismo fin: brindar información, las diferencias consisten en los tipos de información y en los tipos de usuarios. Las bibliotecas, por ejemplo, atienden a un público general o especializado, según se trate de bibliotecas generales, escolares o especializadas, los centros de documentación por su parte, brindan atención a oficinas gubernamentales y a empresas, mientras que los archivos deben informar de manera expedita a los funcionarios de instituciones públicas y privadas para la toma de decisiones, información que tiene la característica de ser única, no se encuentra en libros, revistas, periódicos o cualquier otro material que pueda ser adquirido por medio de la compra, donación o intercambio. Además de ello debe atender a usuarios con diferentes intereses en la investigación. Tal vez por ello, en principio no se consideró a la Archivística dentro de esta armonización, fue hasta 1982, con la publicación del estudio Ramp de la UNESCO, elaborado por Michael Cook, que se planteó de manera más clara, la idea de incluir a la Archivística dentro de la armonización. (Cook, 1986; Fugueras, 1992)

De tal manera que la información que requieren los múltiples tipos de usuarios son cualitativamente distintas, pero utilizan los mismos recursos de transmisión y evaluación de la información. La armonización se refiere entonces a aprovechar las semejanzas y aun las diferencias; armonizar no significa unificar metodologías, tampoco integración de conocimientos, indica interrelación y colaboración. La armonización trata de buscar los aspectos comunes a las Ciencias de la Información con objeto de optimizar los recursos económicos de infraestructura y los intelectuales como los docentes y recursos pedagógicos, pero con énfasis en las características y particularidades de cada disciplina. Así el estudio de Michael Cook, propone un número de asignaturas que son comunes a los profesionales de la información. Algunas de ellas son: Teoría de la Información, Análisis Documental, Estudios de Usuarios, Tecnologías, Conservación, entre otras.

Aún más, el vertiginoso desarrollo de las tecnologías de la información y la comunicación ha propiciado la convergencia de distintos tipos de profesionales especialistas en Informática, Marketing, Comunicación, etcétera.- lo que a su vez ha incidido en la homogeneización de las técnicas de tratamiento documental. Dado que las labores de información dejaron de ser tarea exclusiva de bibliotecólogos, archivistas y documentalistas, las fronteras entre las ciencias informativas han tendido a desvanecerse, ahora existe una amplia cooperación entre ellas, se habla de interdisciplina, multidisclina y transdisciplina, no sólo entre las tradicionales ciencias de la información, sino entre todas aquellas involucradas en el proceso informativo documental.

En 1996 Theo Thomassen volvió a referirse al punto de encuentro entre los archivistas, bibliotecólogos y documentalistas, refiere que es el único grupo de estudiosos que laboran con los productores y los usuarios de la información. En alusión a la ya habitual oposición de los archivistas historiadores a la armonización, expresa que 
la archivística no debe estar subordinada a la Historia ni a las demás ciencias de la información, sino como "unos estudios independientes en el área de las Ciencias de la Información» (Thomassen, 1996)

A pesar de todas las aportaciones sobre la formación armonizada, en México aún no es aceptada del todo esta teoría, hace sólo dos años se creó la carrera de Archivística en la Universidad de San Luis Potosí, donde ya existía la carrera de Bibliotecología e Información. La renuencia se debe en especial a que la Bibliotecología ha tenido un mayor desarrollo: existen asociaciones de bibliotecólogos con una larga tradición; la Archivística por su parte, no ha logrado conformar una agrupación sólida. Todo ello ha incidido para que la idea de la armonización no se haya generalizado.

Por otra parte, el desarrollo de las tecnologías de la información y la comunicación, además de la corriente filosófica llamada postmodernismo, han puesto en crisis los principios básicos de la Archivística. La mayor parte de los archivistas europeos, norteamericanos, australianos, están de acuerdo en que es necesaria la formulación de un nuevo paradigma en Archivística. De acuerdo con Terry Cook la mentalidad posmoderna ha afectado a la Archivística de dos maneras: por una parte, ha influido en la discusión teórica de varias disciplinas como la Crítica Literaria, la Historia de la Literatura, el Psicoanálisis, la Antropología, Cartografía, el Arte y la Archivística. La segunda, reside en su especulación sobre la naturaleza de la historia y otros textos. En efecto, a partir de la publicación del libro de Jacques Derridá, Mal de archivo, los historiadores reflexionaron acerca de la inestabilidad del texto y de la relación que existe entre éste y su autor. Ahora interesa a la historia el análisis de la lengua, los patrones del discurso y de las palabras o del documento, la totalidad del sistema de información, en el contexto de su tiempo y lugar, para evidenciar lo oculto, las motivaciones y las estructuras de poder que dieron origen a los documentos.(Cook, 2000)

El postmodernismo ha puesto en riesgo las nociones de verdad universal o conocimiento objetivo sobre la base de los principios del racionalismo científico de la Ilustración, de tal forma que el empleo del método científico clásico o de la crítica textual, revela lo ilógico de los textos. Por lo tanto, los documentos no pueden separarse de su pasado, nada es neutral, nada es imparcial, nada es objetivo: todo se presenta de la forma en que pretendieron sus creadores, revelan únicamente las relaciones de poder, existentes en la época de que traten los documentos. Así, Jacques LeGoff, dice que el documento no es objetivo, ni inocente materia prima, sino que expresa el control de la sociedad sobre la memoria y sobre el futuro: el documento es lo que queda de las relaciones de poder, es un control de la memoria y por lo tanto de la historia.

Por ello la Archivística debe cambiar, según estos teóricos, la Teoría del Estado, base primordial del principio de procedencia, -pues sólo se conservan los documentos de las instituciones- por sí sola ya no es suficiente ni para la historia ni para la propia disciplina, es preciso volver la mirada hacia lo social, al contexto en que se producen los documentos, ya que éstos son del pueblo y para el pueblo en toda democracia. Si bien la rendición de cuentas, los intereses fiscales y la protección de los derechos de las personas deben primar en todos los archivos, ha de incluirse entre sus funciones ofrecer a los ciudadanos un sentido de identidad, historia, cultura y la memoria personal y colectiva. En pocas palabras, ya no es aceptable limitar la definición de la memoria de la sociedad únicamente a la documentación generada por los Estados. 
De tal manera que el principio de procedencia, el que obliga a respetar la estructura orgánica de la institución generadora de los documentos, sólo responde a la recreación de las relaciones de poder y no a un afán por conservar testimonios de la sociedad. Así por ejemplo la historia de las mujeres ha sido relegada, lo mismo que la historia de los indígenas o de los seres comunes, sobre ellos no existen o hay muy pocos documentos, porque a las instituciones en el poder no les interesa conservar testimonios de ello. En consecuencia, debe reelaborarse el principio de procedencia que respondió a las necesidades decimonónicas y construir uno nuevo basado más bien en el contexto social. (Cook, 2000)

En el plano de la valoración documental, en los Países Bajos se adoptó un método de evaluación de las funciones gubernamentales en lugar de la evaluación de documentos individuales, ahí se hace caso omiso a los principios tradicionales de gestión de archivos y expedientes, que en realidad tienden principalmente a seleccionar y retener la información generada por los procesos administrativos, la estrategia propuesta parte de la evaluación de información, sobre su papel en las actividades del gobierno. A raíz de este planteamiento, se analizan en primer lugar las misiones y tareas necesarias para llevar a cabo las funciones; En general, la información necesaria para reconstruir las funciones esenciales de gobierno es lo que debe mantenerse. El proyecto tiene un alcance más amplio, porque también involucra la interacción del ciudadano con el Estado y el impacto de las acciones del Estado sobre los ciudadanos,

Los archivistas canadienses han dedicado sus esfuerzos precisamente a eso, a repensar el principio de procedencia, desde la perspectiva no sólo de sus creadores, sino también del contexto social. Toda una serie de estudios han florecido para establecer nuevas formas de clasificación documental, en las que se toman en cuenta las acciones, funciones, características físicas de los distintos tipos de documentos, los vínculos horizontales, porque el principio de orden original, por sí solo no responde a las necesidades actuales. (Edwards, 2001)

De acuerdo con Thomasen, el nuevo paradigma de la archivística no es el viejo paradigma, pero lo tiene en cuenta para su formulación, es decir no se parte de cero. Es un nuevo modelo explicativo para el campo científico en una nueva etapa de su desarrollo, un modelo que define los fundamentos de la ciencia y de archivo, pero sólo puede hacerlo sobre la base de las nociones clásicas, en el que las reformula; sus métodos y técnicas seguirán aplicándose en sus ámbitos tradicionales, pero enunciará nuevos principios y métodos. Para afrontar los retos de la postmodernidad, los archivistas deben olvidar su aislamiento y sus nacionalismos para emprender la empresa de manera conjunta, con los investigadores y profesores de todos los países del mundo. (Thomassen, 1999)

Los retos que afronta la archivística giran en torno a las tecnologías de la información y la comunicación, en especial a los documentos electrónicos, a la fiabilidad y autenticidad de su contenido y a su preservación, entre otras cosas. El desafío se agiganta debido a la falta de experiencia consolidada en el entorno tecnológico, a la falta de una total comprensión del continuo periodo de transición tecnológica y a la insuficiencia de un análisis sistemático de los aspectos conceptuales metodológicos. 


\section{Reflexión final}

Consideramos que el modelo kuhniano que se emplea para el análisis de la archivística no es el adecuado. En primer lugar se olvida la indefinición y ambigüedad de lo que es paradigma, cuestión que el mismo Kuhn reconoció. Todos hablan de él, pero nadie sabe exactamente lo que es. Ya en 1965, Margaret Masterman señaló que el término de paradigma era utilizado en la obra de Kuhn con 21 significados distintos. En segundo lugar, según Kuhn, el desarrollo de la ciencia se lleva a cabo como rupturas entre paradigmas, como mutaciones en la forma de hacer ciencia, sin que se puedan comparar, ni decidir cuál es "más verdadera", porque los nuevos conocimientos no reemplazan a la ignorancia sino a otros conocimientos de distinto tipo. Esas rupturas se deben a lo que Kuhn denominó inconmensurabilidad entre paradigmas. Por lo tanto, el hablar de cambios de paradigmas implica una ruptura con la historia pasada, con el capital de conocimientos acumulados, para empezar una nueva historia que nada tiene que ver con la anterior. Se rompe con la tradición científica y se tiene solamente pura innovación.

Ante ese hecho, hemos planteado con respecto al estudio de la Bibliotecología, tal como es entendida en México, semejante a lo que se entiende por Ciencia de Información en el mundo anglosajón y Brasil o Documentación en España, que es mejor utilizar la idea de Lakatos sobre los Programas de Investigación Científica, (Rendón, 2005: 22-25) donde el desarrollo de la ciencia no es el resultado de la confrontación entre una teoría y los hechos; sino de la competencia de dos o más teorías que se confrontan a un mismo hecho. Los programas de investigación científica están constituidos por tres elementos. El primero es el núcleo central que son leyes, conceptos y supuestos fundamentales que le otorgan identidad al programa de investigación y es infalseable. Este elemento proporciona la tradición científica. El segundo componente es el cinturón protector, consistente en hipótesis auxiliares, condiciones iniciales, conceptos y terminología auxiliares, y varias teorías que aunque contrarias, no representan una ruptura total entre ellas, sino que se encuentran bajo la dirección del núcleo duro y son diferentes enfoques que tratan de explicar un fenómeno. Este elemento ofrece la innovación. El tercer componente es la heurística que es el conjunto de reglas metodológicas que indican lo que se debe hacer o está prohibido realizar en el proceso de investigación.

Como resultado de nuestras investigaciones sobre epistemología de la Bibliotecología, hemos propuesto como núcleo central de su Programa de Investigación Científica lo que hemos llamado Sistema Informativo Documental. Dicho sistema está compuesto por la información, el documento, el usuario, la institución informativa documental y el profesional de la información documental y las interrelaciones entre esos elementos. (Rendón: 160-173)

Dentro de ese núcleo central encontramos una ontología común para las disciplinas informativo-documentales: Archivística, Bibliotecología y Documentación. En dicha ontología existen "objetos" compartidos: usuarios, información, documentos, fuentes y fondos de información, institución informativa documental; asimismo se descubren procesos y actividades a las que a todas atañen: el flujo de información, ${ }^{3}$ el ciclo social

${ }^{3}$ El flujo de información documental es el "conjunto de documentos publicados y [...] no publicados [...] que aparecen constantemente y son utilizados en la práctica histórico-social con la finalidad de 
del documento, ${ }^{4}$ la gestión informativa documental, las acciones comunicativas, la administración, entre otras.

Sin embargo, cada disciplina informativa documental posee sus especificidades, sus propios principios, conceptos y manera particular de acercarse a lo general. Por ejemplo aunque clasificar y organizar es común para la bibliotecología y la archivística, esto es, el principio general es válido para $\mathrm{ambas}^{5}$; cada una lo realiza con criterios diferentes.

El reto epistemológico en el análisis de la archivística es por un lado determinar si comparte el núcleo central de su Programa de Investigación Científica junto con las otras disciplinas informativas documentales, o es tan diferente que tiene su propio núcleo central. De la misma manera por ejemplo a la Psicología, que aunque junto con la Medicina o Veterinaria, es Ciencia de la salud, es una ciencia autónoma con su propia identidad y PIC. Sin embargo, aun en este caso de autonomía, es necesario seguir concibiendo el desarrollo de la Archivística como la aparición de diversas teorías (Gestión de Documentos, Archivística Tradicional, Archivística Integral) en el cinturón protector de un mismo PIC, y no de rompimientos paradigmáticos. El anterior enfoque nos proporciona la posibilidad de recuperar la tradición científica acumulada sin negar un sitio para la innovación.

Por otro lado, el segundo reto epistemológico es alejarse de lo concreto, porque esto es cambiante, y de lo pragmático-funcional; para de esta manera construir un cuerpo conceptual de manera más abstracta y general. El afirmar sin más, que el objeto de estudio de la Archivística es el archivo, a semejanza de la Bibliotecología que es la biblioteca, y de la Documentación que es el documento, es simplificar el problema Si se habla de biblioteca, archivo o documento como objetos de estudio, no se conciben éstos como objetos concretos, (al igual que la medicina no estudia los hospitales) sino como abstracciones que implican toda una serie de elementos, procesos y acciones dentro de un sistema de información.

Es más adecuado decir que la Archivística estudia el proceso documental (flujo y ciclo social de la información) dentro del sistema informativo documental que tiene como elementos el archivo, los fondos documentales, su organización, conservación y servicio a usuarios de archivos para la satisfacción de necesidades de información.

Por supuesto que si se afirma que el objeto de estudio de la archivística son los fondos documentales y los archivos, éstos con el devenir del tiempo, van a cambiar. Lo mismo sucede cuando se afirma que el método de la archivística es el tratamiento documental, que consiste en un conjunto de operaciones y tareas que se aplican a los documentos en cada una de sus fases del ciclo vital y que tiene como objetivos

\footnotetext{
intercambiar información.” (Gorkova, 1988: 27)

${ }^{4} \mathrm{El}$ ciclo social de la información incluye la generación, recolección, procesamiento analítico-sintético, almacenamiento, búsqueda y recuperación, diseminación y uso de la información, la cual a su vez lleva de nuevo a la generación de información, repitiéndose de esa manera el ciclo. (Mijailov: 1973, 59-60)

${ }^{5}$ Clasificar es una operación lógica que consiste en agrupar a elementos componentes de un universo en clases excluyentes y complementarias sobre la base de la posesión/carencia de un(os) atributo(s) que constituye el criterio o base de la clasificación. La unión de las clases resultantes de la clasificación es igual al universo; y la intersección de dos clases cualesquiera es vacía.
} 
organizarlos, conservarlos y hacerlos accesibles, manejables y útiles en sus diversos fines. (Duplá del Moral, 1997: 77) En esa cita se observa claramente el nivel pragmático al que se quiere llevar el estudio de la Archivística a expensas del nivel teórico.El reto y objetivo es construir conceptos y nuevas propuestas teóricas, pero sin concebirlas como "revoluciones científicas", sino como nuevas herramientas teóricas (innovación) para estudiar antiguos fenómenos (tradición) que aparecen en contextos novedosos. Esa tarea teórica requiere mudarse de una ontología de primer nivel o otra de un nivel más abstracto. Eso ocurre por ejemplo con la negación del principio de procedencia visto específicamente como origen institucional, para recurrir a un principio más general de contexto.

El afrontar esos retos y cuestionamientos epistemológicos permitirá a la Archivística desarrollarse en el plano teórico, lo que a su vez conlleva en segundo término, a cumplir con su misión práctica, tan importante y necesaria en esta etapa de la sociedad, donde la información ha llegado a devenir en un bien indispensable para poder actuar e insertarse no sólo en la vida económica y científica, sino en la vida política, social, cultural, y todas las esferas de esta sociedad tecnologizada e informatizada.

\section{Referências bibliográficas}

Cook, Michael. 1986 Directrices para la preparación de programas de estudios sobre tecnología de la información para bibliotecarios, archivistas y documentalistas. París, UNESCO.

Cook, Terry. 2000 "Archival Science and Postmodernism: New Formulations for Old Concepts" En:. Archival science, Vol. 1. No.1, pp. 3-24.

"What is past is prologue: A History of Archival Ideas Since 1898, and the Future Paradigm Shift". Archivaria 43 17-63.

Delgado López-Cózar, Emilio.

"Principios, estructura y contenidos de un programa de profesionales de la información y documentación: Propuesta de plan de estudios para la EUBD de Granada”. En: Boletín de la Sociedad Andaluza de de Bibliotecarios. Diciembre de 1992. pp. 4-36.

Duplá del Moral, Ana. (1997). Manual de archivos de oficina para gestores. Madrid: Comunidad de Madrid; Marcial Pons. p. 77.

Edwards, Robert."With Respect to Original Order": Changing Values in Archival Arrangement. AABC Newsletter 11:1(Winter 2001).

Fugueras, Ramón Alberch. "Impacto tecnológico y formación archivística” [En Línea] Disponible en: www.enj.org. [Consultado 24 de julio de 2009]

Fuster Ruíz, Francisco. "Archivística, archivo, documentos de archivo...necesidad de clarificar los conceptos" En: Anales de documentación No. 2. 1999. pp. 103-120

Rendón Rojas, M. A. Bases teóricas y filosóficas de la Bibliotecología. México: UNAM, CUIB. $2^{\text {a }}$ edición. 2005. pp. 22-25.

Shellenberg, Theodore. Archivos modernos: Principios y técnicas, trad. Manuel Carrera E., México, AGN, 1987.

Thoassen, Theo. "Cómo se saca el carnet de conducir en la autopista de la información". En: Actas del XIII Congreso Internacional de Archivos. Beijing, 1996. 
Thomasen, Theo. "The development of archival science and its european dimension" Conferencia de Estocolmo 1999. En Arhol. Archivistika on line, disponible en: http://www.daz.hr/arhol/ thomassen.htm. Consultada el 12 de febrero de 2009. 